\title{
LSST Charge-Coupled Device Calibration
}

\author{
Tiarra Johannas Stout \\ Office of Science, Science Undergraduate Laboratory Internship (SULI) \\ Idaho State University \\ Stanford Linear Accelerator Center Stanford, CA
}

August 10, 2010

Prepared in partial fulfillment of the requirements of the Office of Science, Department of Energy's Science Undergraduate Laboratory Internship under the direction of Deborah Bard at the Kavli Institute for Particle Astrophysics and Cosmology at the Stanford Linear Accelerator Center.

Participant:

Signature

Research Advisor:

Signature 


\section{Table of Contents}

Abstract ....................................................................... 3

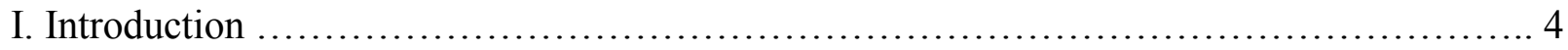

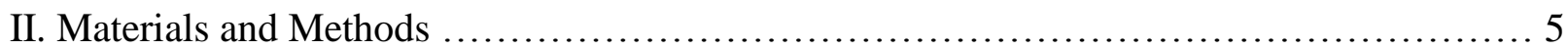

2.1 CCD Chip Calibration ............................................... 5

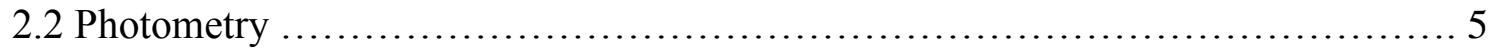

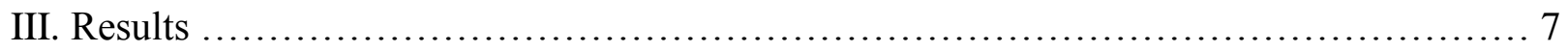

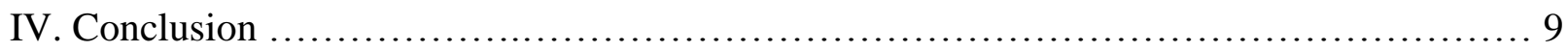

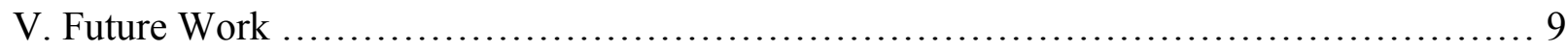

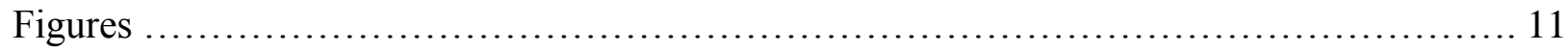

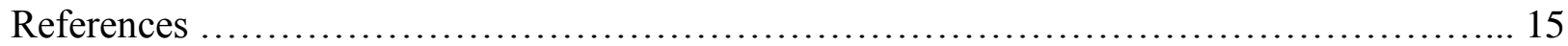




\begin{abstract}
LSST Charge-Coupled Device Calibration. TIARRA JOHANNAS STOUT (Idaho State University, Pocatello, ID 83209) DEBORAH JOANNE BARD (Stanford Linear Accelerator Center, Menlo Park, CA 94025).

The prototype charge-coupled device created at the Stanford Linear Accelerator Center for the Large Synoptic Survey Telescope must be tested to check its functionality and performance. It was installed into the Calypso telescope in Arizona in November of 2008 for this purpose. Since then it has taken many images of various astronomical objects. By doing photometry on standard stars in these images, we can compare our magnitude results to the known magnitudes of these stars. This comparison allows us to then determine the chip's performance and functional capabilities.
\end{abstract}




\section{INTRODUCTION}

Expecting to see first light in 2016, the Large Synoptic Survey Telescope (LSST) is an extremely large ground based telescope that anticipates funding and will be built in Chile. Described as "Wide-Fast-Deep", the LSST will have an unprecedented wide field of view (ten square degrees for surveys), short exposures (fifteen to thirty seconds and still see faint objects), and the largest digital camera in the world [1].

One of the goals hoped to be achieved with this camera is the measurement of dark matter using strong and weak gravitational lensing [1]. Gravitational lensing occurs when a large cluster of galaxies distorts the light from a galaxy behind this cluster. This causes an arc of light to form around the cluster. By measuring the length of this arc, one can calculate how much matter should be present in the cluster. Since the amount that should be present is vastly greater than the amount of visible matter that can be seen, it is postulated that the difference between these two numbers is made up of dark matter. This is a direct way of measuring the amount of dark matter in the universe [2]. Thousands of galaxy clusters will be seen with LSST, allowing precise measurements of strong lensing effects. Weak lensing is a much smaller effect, distorting the shape of galaxies by only a few percent. The scale of LSST will allow these small effects to be measured with a precision unavailable with current smaller surveys.

Some of the other uses for the LSST will be cataloging the entire sky, observing exploding supernovae and near Earth objects, and probing into the nature of dark energy [1].

Since the LSST is such a large project, one organization alone cannot build it. Therefore many organizations have come together, each one working on a specific part of the telescope's construction. Here at the Stanford Linear Accelerator Center (SLAC) the camera is being designed.

Weighing 2800 kilograms ( 6173 lbs), the camera will have approximately 3.2 billion pixels and be 1.6 meters by 3 meters [1]. A charge-coupled device (CCD chip) will be the light 
sensor of the camera. A prototype CCD chip has been constructed and installed in the Calypso telescope in Arizona, where its performance and functional capabilities are being tested.

The LSST CCD array will not be one chip, however, but consist of 189 CCD chips arranged into 21 "rafts." Each raft contains nine CCD chips, which are individually divided into sixteen segments [1]. (See figure 1.)

\section{MATERIALS AND METHODS}

\subsection{Calibration of CCD Chip}

Since being installed in Calypso in November of 2008, the prototype CCD chip has taken numerous astronomical images, which has allowed us to test its capabilities. By doing photometry (the measurement of an object's flux or intensity of electromagnetic radiation) on the standard astronomical objects in these images, we can compare our results using the prototype with known results of these well measured objects. If our results produce the same numbers that have been previously documented, then the CCD chip is performing well. To test the prototype, object EGGR 102 (more commonly known as HIP 66578) was observed and the resulting images were processed.

\subsection{Photometry}

In order to process the images, the program called IRAF (Image Reduction and Analysis Facility) was used. IRAF allows one to manipulate images and convert measurements of star flux from a CCD image to a standard magnitude. It is this standard magnitude that is needed to compare the data taken by the LSST chip to known data of well measured objects.

The first step is to process or clean up the images to remove interference or noise caused by electronic readout noise, thermal electrons, pixel-to-pixel variations, optical non-uniformities, etc, that may later compromise photometry on the actual images [3][4][5]. These effects are removed by taking three types of calibration images, along with science data, throughout the night. 
The first type of image is a bias frame or zero second integration. Using a zero second exposure time to flush and read out the chip, these biases establish the readout noise from the chip [3][5]. For data from the Calypso, a one second "dark" is used for this purpose instead of an actual bias. The same goal is accomplished, however.

The second type of calibration image is called a "dark". These images are taken with the shutters closed to establish the pattern of dark current in the chip [3][4][5]. Dark current is the slight electrical current that still flows through the chip even when no photons are present. This effect, however, is negligible when using an effective CCD chip and short exposure times; therefore, for images taken at Calypso, this is not required.

The third image type is the "flat." There are two kinds of flats: dome flats and sky flats. A dome flat is an exposure taken of a white screen while the dome is still closed. This screen is then illuminated by lamps with a well understood spectrum of light. A sky flat is taken of the sky right as the sun is setting or rising, which gives the sky a uniform illumination. These flats show pixel-to-pixel patterns that result from sensitivity variations in the chip or shadows caused by dust or other obstructions [3][4][5].

After the images are processed, one can then do photometry on the desired objects and extract their "instrumental magnitude", which is the magnitude calculated from the number of photon counts received by the CCD chip. This magnitude comes from measuring the area under the curve of the radial profile of the star, which represents the light from both the star and sky background. (See figure 2) The sky is then subtracted out, divided by the exposure time, and the $\log$ is taken of the final result [3].

However, it is the absolute magnitude of the star that is desired since it can tell us how far away the star is, its composition, etc. Here one must compile a standard star catalog to find the coefficients of the magnitude equations that will be used to convert the instrumental magnitude into a standard magnitude, which is the measurement of an object's intrinsic brightness. If we are 
measuring a well known star, this allows us to compare our results to known results, thereby testing how well our CCD chip is performing.

The catalog format used is from Landolt's UBVRI Standard Stars (2007) of this form:

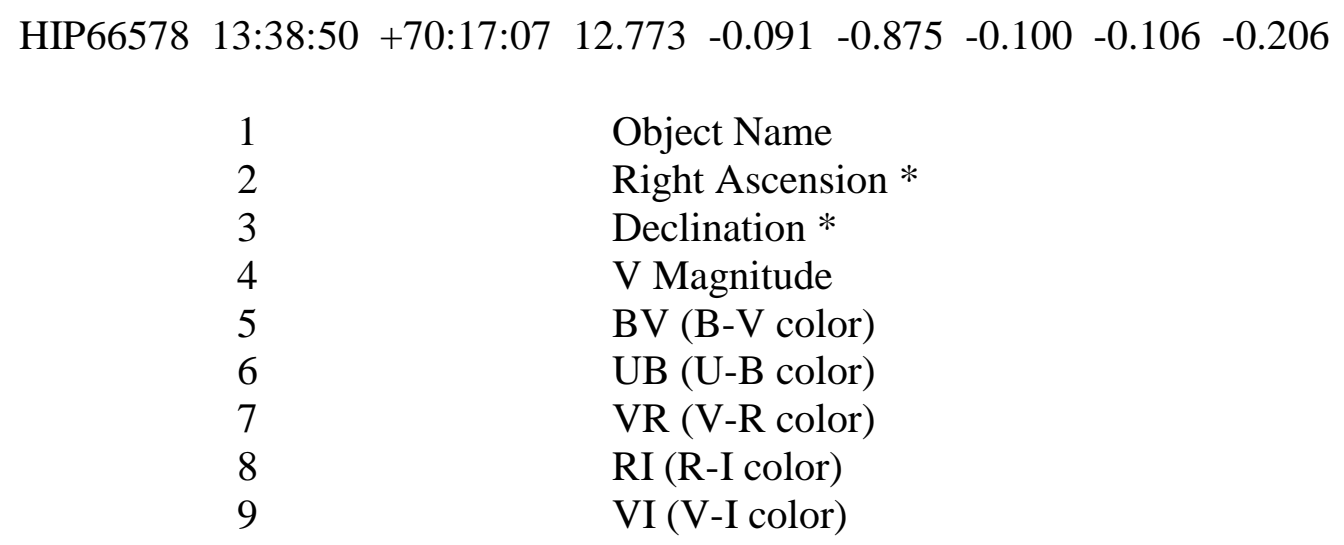

Information for the catalog was taken from the Simbad database.

\footnotetext{
* Coordinates in FK5 coordinate system
} 


\section{RESULTS}

EGGR 102 was measured in the photometric bands i, r, z, and y4, with bands i, z, and y4 being near-infrared filters and $r$ being a visible light filter. Since the standard magnitudes are not known in the $\mathrm{z}$ and $\mathrm{y} 4$ bands, I only tested our results in the $\mathrm{i}$ and $\mathrm{r}$ bands. These are the resulting transformation equations along with their calculated coefficients. (See figures 3 and 4)

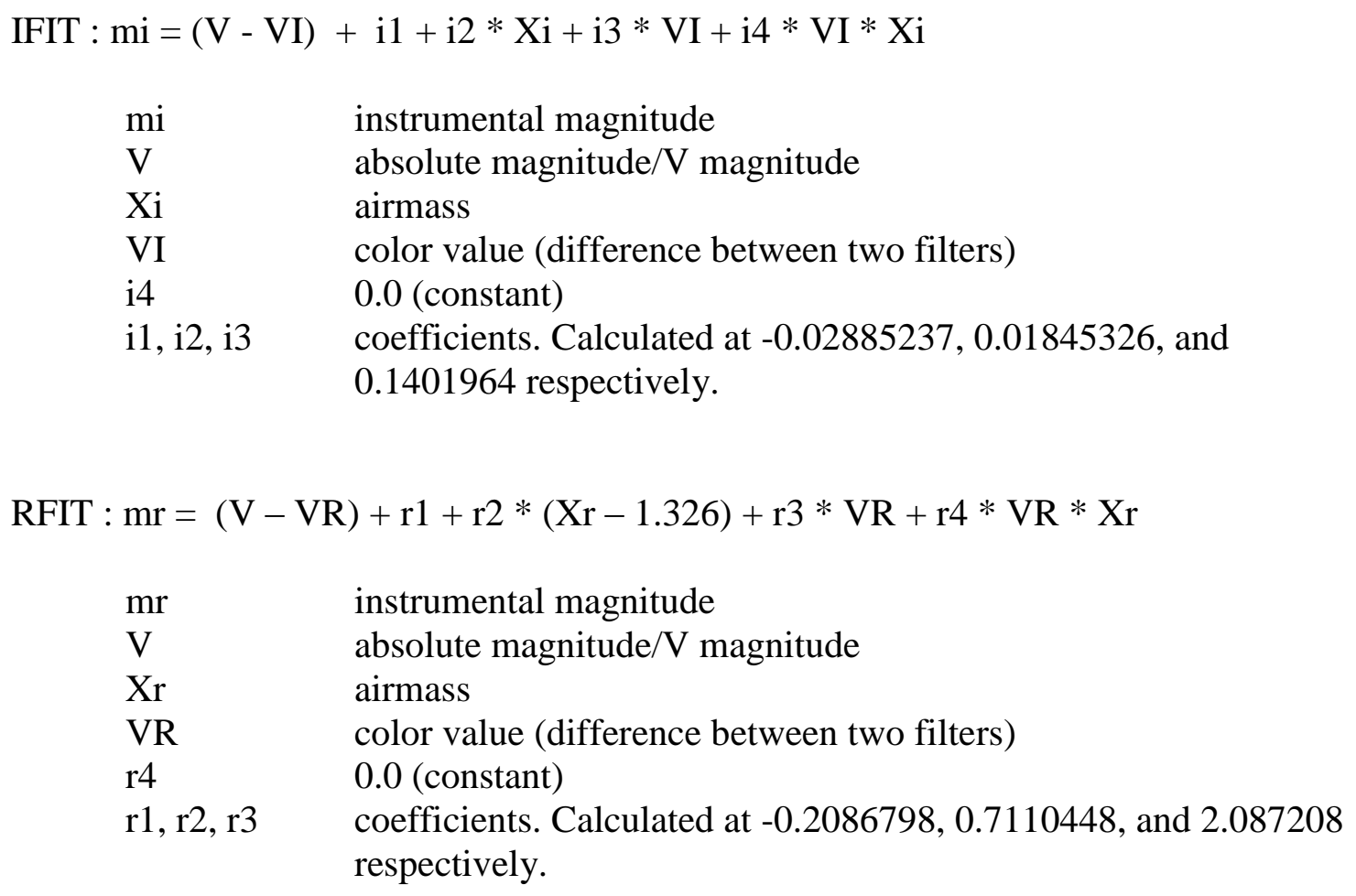

For the IFIT, five datapoints were used in the final fitting, resulting in a root mean square (RMS) of .005886 after deleting an outlier point. The standard magnitude for EGGR 102 in the i filter is 12.979 . Our result was $12.978+/-.006$ after setting the $\mathrm{z}$ magnitude (zero point of magnitude scale) in the IRAF photpars parameter to 23.0429. (See figure 3)

The RFIT solution originally did not converge, therefore I corrected the airmass variable (Xr) by subtracting the average airmass of the images from the airmass variable to bring that term closer to zero to shift the intercept and create a higher precision [5]. After modifying the equation, the RMS equaled .007863 using five datapoints. The standard magnitude is 12.873 with our result being $12.465+/-.008$. (See figure 4) 


\section{CONCLUSION}

Our preliminary results show that the prototype CCD chip is working well and accurately since our results for the absolute magnitude of EGGR 102 are very near that of its known value and the RMS is less than .01 [5]. However, more data is needed to solidly verify its functionality.

The work done here has also revealed a deficiency in the collected data from Calypso so far. Much of the data taken at Calypso is useless for calibration purposes due to flaws in the images, the images not being of standard astronomical objects, or standard objects being measured in filters that do not contain much data. Future images should be taken of standard stars in common filters for calibration purposes, with an attention on image quality.

\section{FUTURE WORK}

Once the calibration is completed, objects with little known data can be observed. New data can also be gathered using the y3 and y4 photometric filters, which were specifically developed by Dr. Kirk Gilmore at the Stanford Linear Accelerator Center (SLAC) for use in the LSST. Further work will also be done on the CCD chip itself, characterizing, and hopefully correcting, the segment variations caused by different zero-point biases, thereby giving the image more uniformity.

\section{ACKNOWLEDGEMENTS}

I would like to thank my mentor Dr. Deborah Bard for her support, willingness to answer my questions, and desire to help me succeed during my time here at SLAC. I would like to thank Dr. Kirk Gilmore as well for his support and direction while my mentor was out of the country. SULI is an extraordinary program, and director Dr. Steve Rock was very helpful throughout the entire experience. However, my greatest thank you goes out to the DOE for funding opportunities like this. Thank you. 


\section{FIGURES}

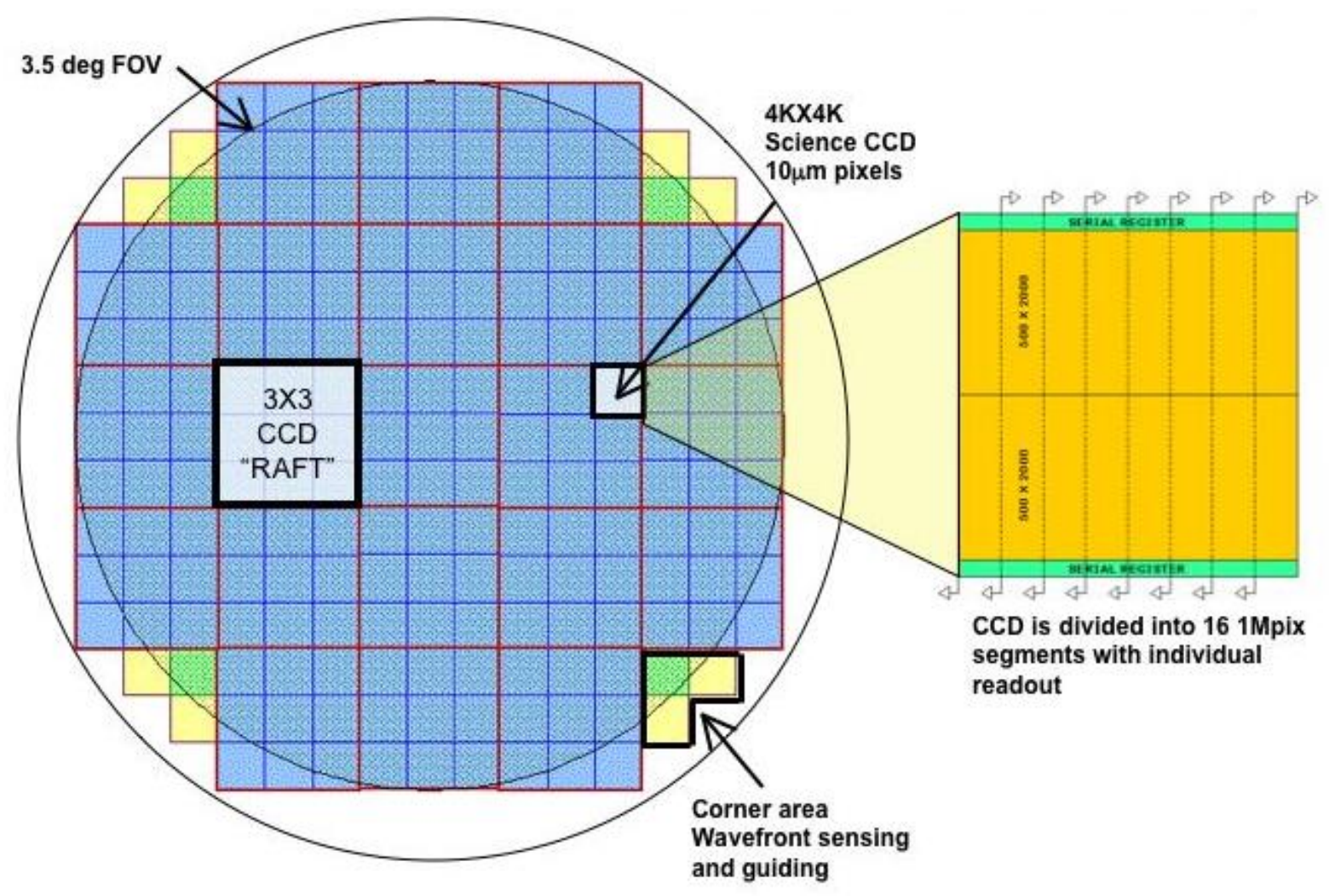

Figure 1: LSST CCD Array. Courtesy of www.lsst.org 


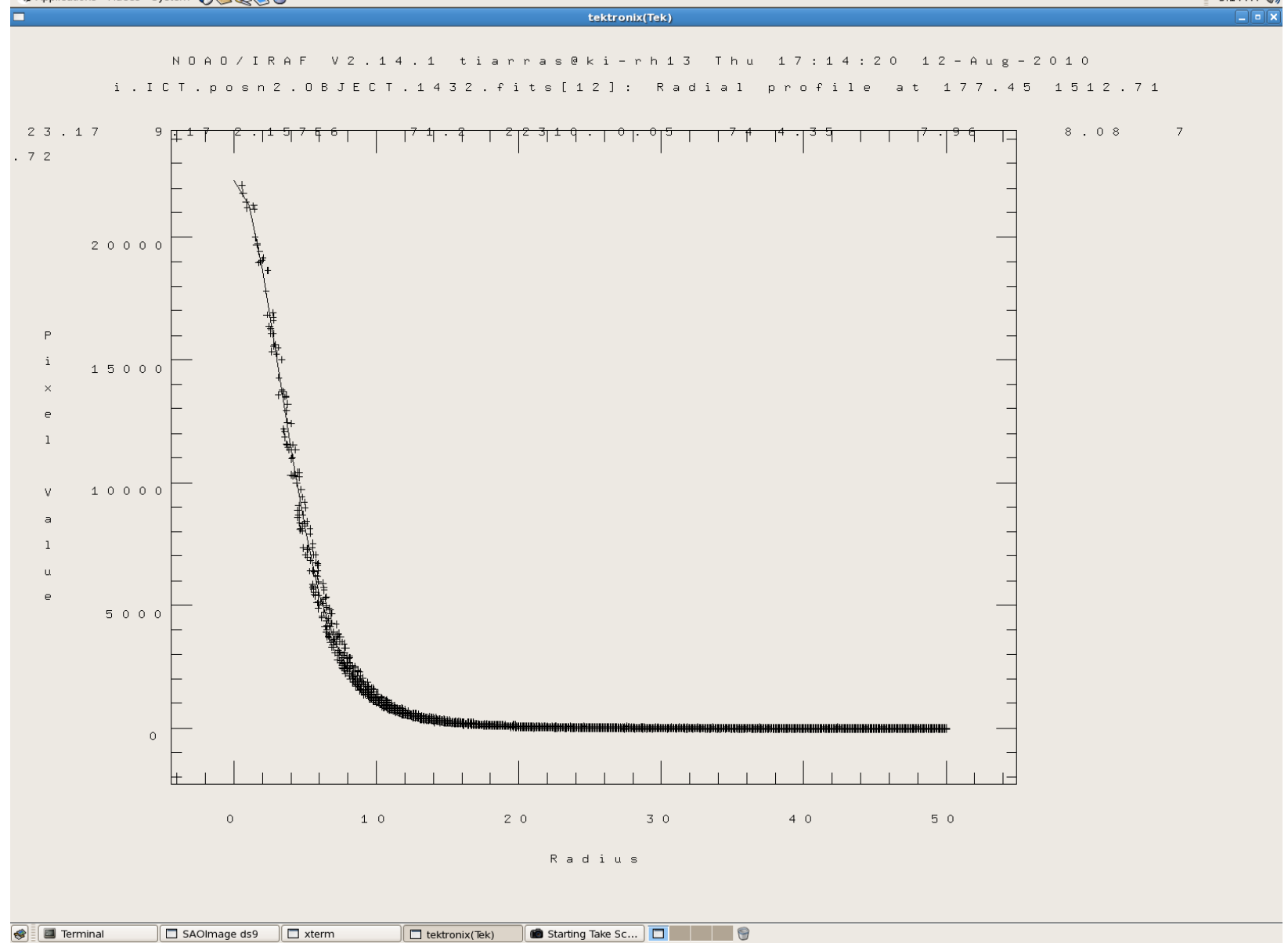

Figure 2: Example of a star radial profile. Produced by Tiarra J. Stout using IRAF and object EGGR 102. 


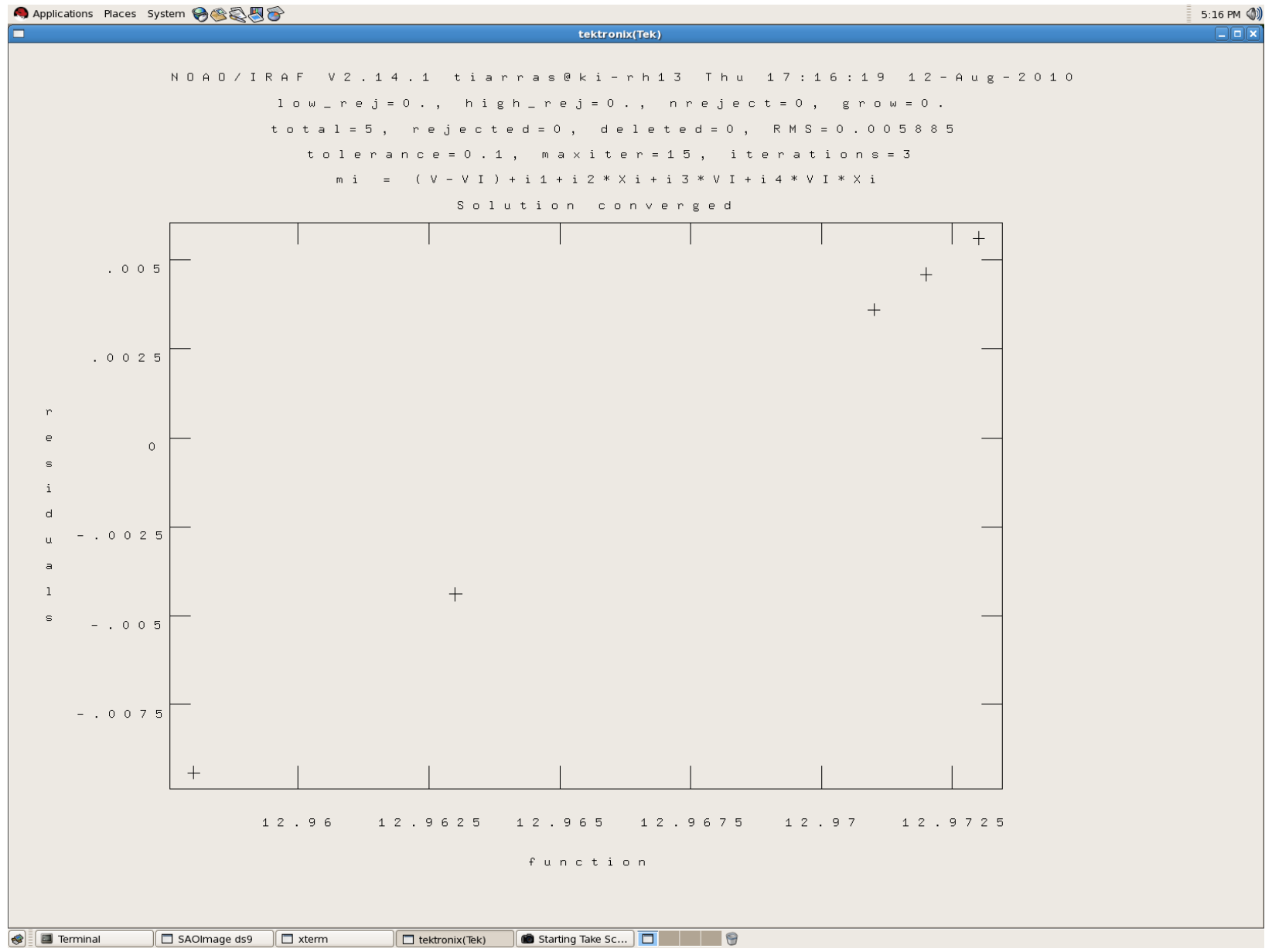

Figure 3: Result of fitting to solve for magnitude coefficients in the $\mathrm{i}$ band. The $\mathrm{y}$-axis shows residuals (the error between the fitted magnitudes and the measured magnitudes). The $\mathrm{x}$-axis shows the function, which is the fitted magnitude. Produced by Tiarra J. Stout using IRAF. 


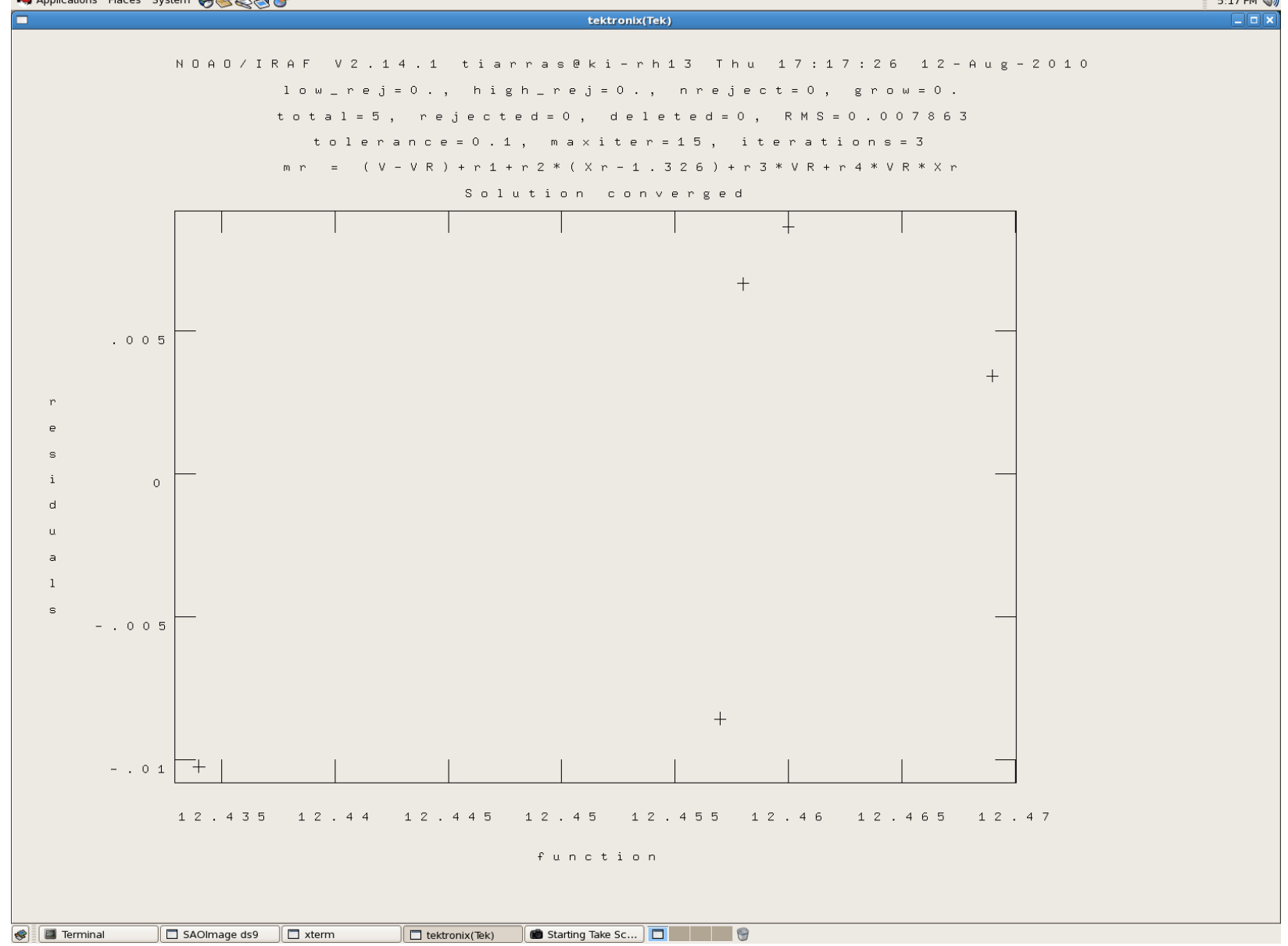

Figure 4: Result of fitting for magnitude coefficients in $r$ band. Produce by Tiarra J. Stout using IRAF. 


\section{REFERENCES}

[1] "Large Synoptic Survey Telescope.” Internet: http://www.lsst.org/lsst, August 13, 2010 [July 10, 2010].

[2] Bartelmann, Matthias, et al. "Weak Gravitational Lensing." Physics Report, vol. 340, pp. 291-472, Dec 1999.

[3] Marschall, Laurence A. "CCD Photometry using IRAF at Gettysburg College and the national Undergraduate Research Observatory." Internet: http://www.nuro.nau.edu/info/iraf.htm, [June 25, 2010].

[4] Massey, Philip, et al. "A User's Guide to Stellar CCD Photometry with IRAF." Internet: http://iraf.net/irafdocs/daophot2/, April 15, 1992 [June 25, 2010].

[5] Grisetti, Robert. "A Student's Guide to CCD Photometry Data Processing using IRAF at the University of Central Florida." Internet:

http://www.physics.ucf.edu/ yfernandez/iraf/ourmanual.pdf, July 3, 2006. [July 10, 2010]. 